\title{
Website www.emergencyprotocol.net to Support Prevention of Metabolic Emergencies in Patients with Hepatic Glycogen Storage Diseases and Fatty Acid Oxidation Disorders
}

Journal of Inborn Errors of Metabolism \& Screening 2021, Volume 9: e20210025 DOI: https://doi.org/10.1590/2326-4594JIEMS-2021-0025

\author{
Carolina F. Moura de Souza' (D), Bibiana M. de Oliveira',2 (D, \\ Ida V D. Schwartz ${ }^{1,2}$ and Terry Derks ${ }^{3}$
}

Keywords: Glycogen Storage Disease, Glycogenosis, Fatty acid oxidation defects, Inborn errors of metabolism, Emergency management.

Hepatic glycogen storage diseases (GSD) and fatty acid oxidation defects (FAODs) are rare diseases in the group of inborn errors of metabolism (IEM). Patients with such diagnoses are susceptible to life-threatening decompensations in catabolic states, such as fasting, infections, fever, and vomiting [1,2]. Under these conditions, treatment must be given quickly and accurately in order to prevent and reduce morbidity and mortality $[1,3]$.

Generators of IEM-specific emergency letters are available in some languages, as recently summarized by Rossi and coworkers $[1,4]$. The purpose of this letter is to present the first automated generator of emergency letters for the management of hepatic GSD and FAODs available in multiple languages, including Portuguese.

The question "How should sickness and emergency situations be managed for patients with liver GSD?" was recently prioritized in the international priority setting partnership [5]. Within the CONNECT MetabERN project, IEM experts and patient organization representatives from many countries have collaborated to improve and expand emergency letters and their availability for health care providers and patients $[1,4]$. The design of the protocol and translation into Portuguese had the participation of our team with experience in managing these clinical conditions.

By accessing https://www.emergencyprotocol.net/Generator/ Disclaimer, personalized emergency letters can be generated automatically for patients with FAODs (MCADD, MADD, VLCADD, LCHADD, and MTP deficiency) and GSD (type 0, Ia, Ib, IIIa, IIIb, IV, VI, IX, XI, and undefined).
The rationale for this protocol is multiple:

- The key measure in many IEM emergency protocols is the prevention of catabolism and subsequent metabolic decompensation by promoting anabolism [1,2];

- When GSD or FAOD patients become sick with for instance fever and vomiting, the families take care of the first emergency measures starting at home;

- When patients need to be admitted to the hospital, the first health care professionals in line usually are no metabolic specialists or GSD experts, and

- It is important that everybody feels supported by uptodate medical information and possibilities for good communication.

The following data are required to generate the emergency letter: type of GSD or FAOD, patient date of birth, body weight (in $\mathrm{kg}$ ), language (Portuguese), and name of the center where

${ }^{1}$ Hospital de Clínicas de Porto Alegre, Serviço de Genética Médica, Rio Grande do Sul, Porto Alegre, Brazil.

${ }^{2}$ Universidade Federal do Rio Grande do Sul, Programa de Pós-Graduação em Genética e Biologia Molecular, Rio Grande do Sul, Porto Akegre, Brazil. ${ }^{3}$ University of Groningen, University Medical Centre Groningen, Beatrix Children's Hospital, Section of Metabolic Diseases, Groningen, The Netherlands.

Received June 10, 2021, and in revised form July 18, 2021. Accepted for publication July 22, 2021.

\section{Corresponding Author:}

Carolina F Moura de Souza, Email: cfsouza@hcpa.edu.br 
follow-up occurs. The information provided will not be saved or stored anywhere.

This tool was designed to assist pediatricians, general practitioners, medical specialists, nurses, patients, and their caregivers to generate a personalized emergency letter for both home and in-hospital management of metabolic emergencies that indicates the dose of oral and intravenous solutions to be used according to the patient's body weight and which tests should be ordered on admission in each condition. This tool, carefully developed by a team of international and Brazilian experts, facilitates and provides access to best management practices within what is considered the current best practice for patients with hepatic GSD or FAODs. Each emergency letter should be carefully reviewed by the person who generated it and should not be considered a substitute for individualized advice from a genetic/ metabolic disease expert or the patient's attending physician.

Patients with IEM such as GSD and FAODs are at risk of potential life-threatening acute metabolic decompensations [2]. National emergency management protocols are scarce in Brazil. However, they are essential to save the lives of these patients in the presence of metabolic decompensation. As Brazil is a country of continental dimensions, with few experts in medical genetics and IEM [6], such protocols can facilitate the acute management of complex cases where simple measures can save lives. Metabolic centers in Brazil who provide 24/7 medical care to IEM patients are invited to share their contact details to info@stofwisselingsziekten. $\mathrm{nl}$ and hence, to use http://www.emergencyprotocol.net [7] to generate emergency letters for their patients.

\section{Funding}

The author(s) received no financial support for the research, authorship, and/or publication of this article.

\section{Authors' Contributions}

BMO and CFMS wrote the manuscript. TD and IVDS participated in critical analysis and review of the manuscript.

\section{Declaration of Conflicting Interests}

The authors declare to have no conflicts of interest.

\section{References}

1. Hoogeveen IJ, van Amerongen T, Weynschenk FJ, et al. A generic emergency protocol for patients with inborn errors of metabolism causing fasting intolerance: a retrospective, single-center chart review of 128 patients. Res Sq. $2020 \mathrm{Feb}$ 06. doi: $10.21203 /$ rs.2.22763/v1

2. Schwartz IV, de Souza CFM, Giugliani R. Tratamento de erros inatos do metabolismo. J Pediatr (Rio J.). 2008;84 (4 Suppl):S8-S19. doi: 10.1590/S0021-75572008000500003

3. Hawkes CP, Walsh A, O'Sullivan S, Crushell E. Doctors knowledge of the acute management of Inborn Errors of Metabolism. Acta Paediatr. 2011;100(3):461-463. doi: 10.1111/j.1651-2227.2010.02062.x

4. Rossi A, Hoogeveen IJ, Lubout CMA, et al. A generic emergency protocol for patients with inborn errors of metabolism causing fasting intolerance: a retrospective, single-center study and the generation of www. emergencyprotocol.net. J Inherit Metab Dis. 2021 Apr 12;1-12. doi: 10.1002/jimd.12386

5. Peeks F, Boonstra WF, de Baere L, et al. Research priorities for liver glycogen storage disease: an international priority setting partnership with the James Lind Alliance. J Inherit Metab Dis. 2020;43(2):279-289. doi: 10.1002/jimd.12178

6. Giugliani R, Vairo FP, Riegel M, et al. Rare disease landscape in Brazil: report of a successful experience in inborn errors of metabolism. Orphanet J Rare Dis. 2016;11:76. doi: 10.1186/s13023-016-0458-3

7. Metabolic emergency protocol. https://www. emergencyprotocol.net/Generator/Disclaimer. Accessed: May 20, 2021. 